\title{
Advances in Optical Components and Subsystems for Wavelength-Division Multiplexing Communications
}

\author{
Abdul Ahad S. Awwal, FELLOW SPIE \\ Lawrence Livermore National \\ Laboratory \\ NIF Programs/NIF Project/Systems \\ Engineering \\ L-495, P. O. Box 0808 \\ Livermore, California 94551 \\ E-mail: awwal1@1lnl.gov \\ Achyut K. Dutta, FELLOW SPIE \\ Banpil Photonics, Inc. \\ 1299 Parkmoor Avenue \\ San Jose, California 95126 \\ E-mail: akdutta@banpil.com
}

The implementation of optical networks is considered to be the only solution to meet the future demands in the communications area. Despite the economic downturn, we received an encouraging level of submissions for this special section. Some of the papers included here were initially presented in the SPIE Information Technologies and Communications (ITCOM) conference on active and passive components and also in the optical computing algorithms and architecture conference. This special section is comprised of 14 papers in the areas of wavelengthdivision multiplexing (WDM) components, systems and optimization, and applications.

Between the source and the detector of an optical network, there are a number of important components technologies that make up the optical WDM network. The WDM components papers included in this special section are on interconnections, waveguides, fiber amplifiers, dispersion compensators, switching, add-drop multiplexers, clock recovery circuits, and detectors. In the first paper of this series, Kawai discusses interconnections between vertical-cavity surface emitting lasers (VCSEL) sources and fibers. Various alignment and packaging issues for developing interconnections between VCSEL sources and fiber waveguides are addressed in this paper. Waveguides consisting of heterostructured photonic crystals are the topic of the next paper by Ohtera et al. The authors describe an autocloning technology that minimizes the propagation loss to $0.1 \mathrm{~dB} / \mathrm{mm}$. To reduce the propagation loss while increasing the capacity and distance of an optical network, laser amplifiers are essential. In the next paper, Wang and Dutta present a model for an Er-Yb double-clad amplifier. Theoretical as well as numerical analysis of amplifier gains for various pump and signal powers are presented. One of the critical components for achieving high-bit-rate communications systems is polarization mode dispersion (PMD) compensation. In the next paper, Khosravani quantifies the effect of PMD on system performance. The author presents ways to compensate for the PMD and minimize signal distortion. Distortion may degrade the performance of optical switching devices. In the next paper, by Pérennou et al., cross talk performance of an acousto-optic switch caused by distortion induced by multiple modulation signals is analyzed. They show a degradation of signal-to-noise ratio caused by these intermodulation effects.

The next three papers address components at the receiving end of the transmission system. Resonances characterized by maximum transmission/absorption have been used successfully for creating passive optical components. The paper by Işçi et al. uses a microsphere resonator to produce dips in the transmission characteristics, which can subsequently be used to construct an add/drop multiplexer. Add/drop multiplexers and demultiplxers at the receiving end need a stable clock source to operate. The next paper by Zhu et al. presents a method of clock recovery using a phase-locked loop in a Mach-Zehnder modulator. They show that it is possible to recover a 10$\mathrm{GHz}$ clock from a 40- and $80-\mathrm{Gb} / \mathrm{s}$ data signal with only 50 -fs jitter. Demultiplexer design using phased arrays is the topic of the last paper in the component category by Yehia and Khalil. Cascading small multimode interference phasar devices with high uniformity, they optimize a large structure with very low nonuniformity (less than 0.2 $\mathrm{dB})$. 
The second area on systems and optimization includes four papers that span from metropolitan WDM to optimization of various cost functions. DeCusatis reports results from DWDM test beds for metropolitan area data communication networks. He evaluates the performance in terms of scalability and performance for different data communication protocol. Katangur, Pan, and Fraser apply simulated annealing to minimize the number of passes to avoid cross talk for a WDM network. They compare the performance of routing and scheduling using their method with several other methods including the genetic algorithm. Kartalopoulos proposes a WDM transmission method supporting many clients with different traffic types, which may be more than the number of wavelengths. He also discusses strategies to optimize cost and enhance quality of the network. Network cost optimization is the topic of the next paper by Billah, Wang, and Awwal. They propose algorithms for grooming the traffic to minimize the number of add/drop multiplexers. Their approach provides more general results than have been obtained previously.

In the last section, we see two different applications of WDM components in the arena of digital optical computing, namely digital memory logic and parallel computing architecture. McAulay provides an architecture for constructing basic memory elements such as a flip-flop using a semiconductor optical amplifier. The simulation shows more than $5-\mathrm{Gb} / \mathrm{s}$ operation as well as bistable behavior essential to achieve memory logic. In the last paper of this special section, the applications of optical communication components to digital computing or smart pixel arrays, characterized by having optical I/O with digital intelligence in each pixel, are considered. Mal et al. develop and compare two different general purpose architectures of smart pixel computing. Their architecture based on CMOS technology, reduced instruction set computer architecture, and photoreceiver circuits has the potential to be used for page-oriented data processing applications.

The balance of the papers in this special section weigh heavily toward the components side. As the components mature, more systems-level testing will be on the horizon. Interesting applications are expected to spring up as researchers from different areas look for devices elsewhere to fit into their applications.

We would like to thank all those who made this special section on WDM components, systems, and applications possible-especially the authors for all the submitted papers and the reviewers for their time and effort. We would also like to thank Karolyn Labes and other SPIE staff for working with us to make this special section a reality.

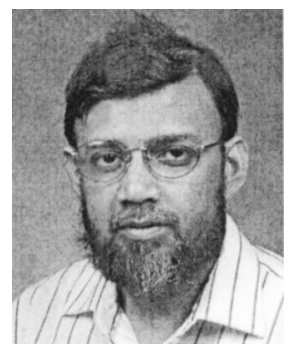

Abdul Ahad S. Awwal currently is with the Systems Engineering group at the National Ignition Facility of the Lawrence Livermore National Laboratory (LLNL). From 1989 to 2001, he was with Wright State University in the Computer Science and Engineering Department. He joined LLNL in 2002. He works on a variety of research areas including automated optical alignment, pattern recognition, adaptive optics, digital optoelectronic computing, computer arithmetic, optical/digital image processing, neural networks, electro-optic displays, optical systems design, machine vision, optical sensors, and digital systems design. He has published refereed articles in numerous journals as well as international conferences. $\mathrm{He}$ is the author of more than 150 published articles including 65 articles in refereed journals, a textbook on optical computing (John Wiley \& Sons, 1992), and three book chapters. In 2003, he received an R\&D 100 award with his team for developing an adaptive optics phoropter. He received the Excellence in Teaching Award in 1996 from Wright State University for distinguished teaching in the College of Engineering and Computer Science. He has served as a special guest editor for several journals. Dr. Awwal is a fellow of SPIE, a member of OSA, and a senior member of IEEE. He has served as a co-chair for SPIE conferences on photonic devices and algorithms for computing since 1999 and for ITCOM conferences on active and passive components for WDM Communications since 2001.

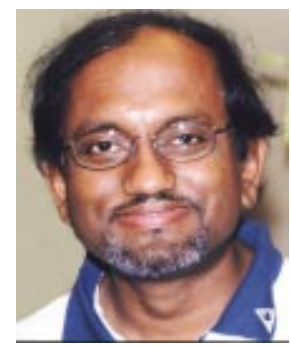

Achyut K. Dutta received his BS degree in electrical and electronics engineering from the Bangladesh University of Engineering and Technology, Dhaka, Bangladesh, in 1984, and the MS and PhD degrees both in electronics engineering from Shizuoka University, Japan, in 1988 and 1990, respectively. From 1985 to 1990 , he was a research associate at the Research Institute of Electronics, Shizuoka University, Japan, where he worked on high-speed position-sensitive optical detectors and amorphous silicon-based avalanche photodiode detectors. From 1991 to 1997, he was a senior research scientist at NEC Central Research Lab., where he was responsible for many projects and contributed to many optical components for general and communication applications. His research and development accomplishments include high-brightness LEDs, laser diodes, high-speed VSCELs, silica waveguides on Si wafers using APCVD technology, transceivers for plastic optical fiber (POF) links, and interconnects and packaging. From 1997 to 2002, he was a senior engineering design manager at Fujitsu, USA, where he started the high-speed module design center and was responsible for the development of high-speed (from 2.5 to $40 \mathrm{~Gb} / \mathrm{s}$ ) laser diode, modulator, and photodetector module products and transferring the technology to manufacturing in Japan. He first introduced the embedding technique for optical and microwave packaging and designed the optical subsystems for short- and long-haul optical communication. In 2002, he founded Banpil Photonics, based in Silicon Valley, California, where he is the president and chief technology officer. He has published more than 100 technical papers and four book chapters and has edited/authored four books (three published by Academic Press, Boston, MA) and five conference proceedings. He holds twenty-two patents on optical components, subsystems, and related technologies. He has served as general chair, organizer, conference chair, program chair, and technical program committee member of numerous conferences for the Optical Society of America (OSA) and SPIE. He is a fellow of SPIE and a senior member of IEEE. He received NEC's best R\&D Award in 1996 for his innovative contributions in POF data links, and was also selected as one of three finalists for the 1996 IEEE LEOS engineering award. He was also awarded a Japanese Ministry of Education Scholarship from 1985 to 1991 for his postgraduate studies. 